\title{
Inhibition of Iodine Organification and Regulation of Follicular Size in Rat Thyroid Tissue In Vitro
}

\author{
Christine Glaser, ${ }^{5}$ Ulrich Marti, ${ }^{1,2}$ Mary Elizabeth Bürgi-Saville, ${ }^{1}$ Charles Ruchti, ${ }^{4}$ \\ Mathias Gebauer, ${ }^{3}$ Markus W. Büchler, ${ }^{5}$ Hans Gerber, ${ }^{2}$ Ulrich Bürgi,, and Hans-Jakob Peter ${ }^{3}$ \\ ${ }^{1}$ Endocrine and Diabetes Division; ${ }^{2}$ Department of Clinical Chemistry; ${ }^{3}$ Division of Medicine, Anna Seiler; \\ ${ }^{4}$ Department of Pathology, ${ }^{5}$ Department of Visceral Surgery, University Hospital, Bern, Switzerland
}

The factors mediating the accumulation of thyroglobulin are of great importance to the understanding of the pathogenesis of human and experimentally induced colloid goiters. To elucidate further the underlying cellular mechanism, thyroid fragments from newborn rats were incorporated into semisolid alginate beads and were cultured as three-dimensional organoids for up to $21 \mathrm{~d}$. In five parallel cultures, the medium contained either no supplements (group A), Nal (group B), thyroid-stimulating hormone (TSH) (group C), Nal plus TSH in the same concentrations as $B$ and C (group D), or Nal and TSH (as in group D) plus methimazole (MMI, group E). The thyroid organoids maintained morphological integrity, functional activity, and ability to proliferate in vitro. Addition of iodine to the cultures significantly increased mean $( \pm$ SEM) follicular diameters from $19.5 \pm 0.7 \mu \mathrm{m}$ in controls to $33.9 \pm 2.2 \mu \mathrm{m}(p<0.0001)$ when $\mathrm{Nal}$ was added alone (group B), and $30.4 \pm 1.7 \mu \mathrm{m}(p<0.0001)$ when combined with TSH (group D). The effect of Nal on follicular size was abolished by MMI (group E, follicular diameter $23.5 \pm 1.3 \mu \mathrm{m}$ ). The results presented support the recent finding, using a rat colloid goiter model, that not only TSH but also iodine organification or its inhibition are important factors in modulating follicular morphology.

Key Words: Follicular diameter; alginate culture; thyroid gland; baby rat.

\section{Introduction}

The pathogenesis of colloid goiter has been a subject of debate for many years. In the 1920 s, it was proposed that

Received June 29, 1999; Revised July 26, 1999; Accepted July 26, 1999.

Author to whom all correspondence and reprint requests should be addressed: U. Marti, Endocrine and Diabetes Division, University Hospital Bern, Freiburgstrasse 15, CH-3010 Bern, Switzerland. E-mail: ulrich.marti@dkf2.unibe.ch colloid-rich goiters developed in hyperplastic glands, after the removal of the goitrogenic stimulus $(1,2)$. This concept was generally accepted for $50 \mathrm{yr}(3,4)$. However, it explained neither the observation that colloid accumulation occurs in most goiters exposed to chronically increased thyroid-stimulating hormone (TSH) (which is still a causative factor of many endemic goiters) nor the coexistence of microfollicular areas containing little colloid and macrofollicular colloid-rich areas in simple goiters (5-8).

Later, it became apparent that the supply of thyroid iodine was also an important factor in the development of colloid goiters. It was reported that thyroglobulin accumulated in the thyroid glands of rats and mice injected with TSH and, at the same time, fed a high iodine diet (9). Furthermore, it could be shown that colloid goiters could be induced in rats and mice by treatment with 5,5-diphenyl-2thiohydantoin (DPTH) (10). This substance raises TSH by increasing fecal loss of thyroid hormones and inhibiting the conversion of thyroxine $\left(\mathrm{T}_{4}\right)$ to triiodothyronine $\left(\mathrm{T}_{3}\right)$, without influencing iodine uptake/organification by the thyroid gland $(3,11)$. By contrast, when iodine organification was blocked by methimazole (MMI) in DPTHtreated rats, hyperplastic, microfollicular goiters developed (10). These reports clearly established the idea that colloid accumulation not depends on intense TSH stimulation but also on thyroidal iodine uptake.

The extracellular matrix (ECM) and its specialized form, the basement membrane (BM), play very important roles in the replication, organization and biological function of cells (for a review, see ref. 12). Therefore, it would seem possible that variations in the ECM/BM might be involved in disease states of tissues, particularly those in which alterations in tissue structure are involved, e.g., in goiter or tumor growth. The ECM/BM in the thyroid has received relatively little attention. Recently, we described the distribution patterns of various ECM/BM components (laminin, collagen IV, perlecan, fibronectin, osteonectin and some laminin variants) in normal and toxic adenomatous human thyroid tissue (13). Although there were no striking 
Table 1

Mean Follicular Diameters $( \pm$ SEM)

\begin{tabular}{lccccc}
\hline & \multicolumn{5}{c}{ Group } \\
\cline { 2 - 6 } & $\mathrm{A}$ & $\mathrm{B}$ & $\mathrm{C}$ & $\mathrm{D}$ & $\mathrm{E}$ \\
\hline Treatment & Control & $\mathrm{NaI}$ & $\mathrm{TSH}$ & $\mathrm{NaI} / \mathrm{TSH}$ & NaI/TSH/MMI \\
$n$ & 124 & 136 & 58 & 146 & 103 \\
Follicular & & & & & \\
diameter $(\mu \mathrm{m})$ & $19.5 \pm 0.7$ & $33.9 \pm 2.2^{a}$ & $23.6 \pm 1.4^{b}$ & $30.4 \pm 1.7^{a}$ & $23.5 \pm 1.3^{b, c}$ \\
\hline${ }^{a}{ }_{p}<0.0001$ vs control. & & & \\
${ }_{b} p<0.0001$ vs B. \\
${ }^{c} p<0.0001$ vs D. (All other statistical differences are not significant.)
\end{tabular}

differences in the composition of the ECM/BM in the adenomas studied, there was a quite marked production of fibronectin in the capsules surrounding the adenomas (13).

In a further report, we were able to show the ability of the alginate gel culture system to retain ECM and follicular structure of rat thyroid tissue. In addition, iodine organification was maintained in the cultured organoids (14).

The present study was undertaken with two aims: first, to confirm the influence of iodine supply on colloid accumulation previously demonstrated in vivo $(9,10)$ in a defined in vitro model using thyroid fragments from newborn rats; and, second, to study the distribution patterns of some ECM/BM components under different experimental conditions.

\section{Results}

\section{Morphological Findings}

Table 1 summarizes the measurements of follicular diameter from the five groups studied. Follicular diameter was significantly increased in groups $\mathrm{B}(\mathrm{NaI})$ and $\mathrm{D}(\mathrm{NaI} /$ $\mathrm{TSH}$ ). This increase in follicular diameter was apparently inhibited when iodine uptake by the cells was blocked by the addition of MMI to the culture medium (group E). These differences in follicular size are illustrated in Fig. 1, which shows immunohistochemical staining for thyroglobulin in the five groups. In cultures treated with $\mathrm{NaI}$ (group B) or NaI plus TSH (group D), large follicles are present filled with thyroglobulin containing colloid. In group E, which was treated with MMI, the follicles are small and the colloid is almost absent.

BM components collagen IV, fibronectin, perlecan, and laminin were investigated by immunohistochemical staining; Fig. 2 shows an example of the results obtained for laminin. Staining for this BM component was observed around individual follicles in all five groups, and there was no apparent difference in the intensity of the staining among the groups. Similar results were found for the other BM components (data not shown).

5-Bromo-2-deoxyuridine (BrdU) incorporation and immunostaining showed that all groups contained dividing cells (data not shown).

\section{Discussion}

Some years ago, in an in vivo model, it was shown that DPTH induces large colloid-rich goiters by increasing fecal loss of thyroid hormones and inhibiting conversion of $\mathrm{T}_{4}$ to $\mathrm{T}_{3}(10)$, thereby increasing endogenous TSH and inducing goiters without impairing iodine binding. DPTH goiters treated with MMI were microfollicular, although serum TSH was increased to the same level as in the goiters treated with DPTH alone. These findings supported our hypothesis that the degree of iodine organification and not TSH is the decisive factor in determining thyroid follicular size $(9,10)$.

The results of the present study confirm our earlier in vivo experiments $(9,10)$. Contrary to the classical concept (2) that colloid goiters are only produced from originally hyperplastic microfollicular goiters when the goitrogenic stimulus ceases, we have shown, in vitro, that elevated TSH and colloid accumulation are not mutually exclusive effects. Tissue treated with iodine (group B) has follicular diameters nearly twice as large as those of the control group (Table 1). The simultaneous exposure of the tissue to TSH and iodine (group D) demonstrates that TSH has no additional effect on the follicular diameters. However, the inhibition of iodine organification by MMI reverses the iodine-induced increase in follicular diameter (group E). TSH alone dose not significantly affect the follicular diameter (group C). The BrdU incorporation experiment confirms that the cells, in all groups, are dividing in the alginate beads. It is now clear that iodine organification or its inhibition is also an important factor influencing whether thyroid tissue becomes micro- or macrofollicular.

Immunohistochemical studies on the basement membrane components laminin, fibronectin, perlecan, and collagen IV have confirmed their presence and normal distribution in the alginate-embedded tissue. However, there was no obvious difference between the groups. Although iodine can affect follicular size, these results suggest that none of the culture media supplements used in this study affect the BM composition.

This in vitro model will facilitate further investigations into the regulation of follicle size and thyroglobulin accumulation, under defined in vitro conditions. 

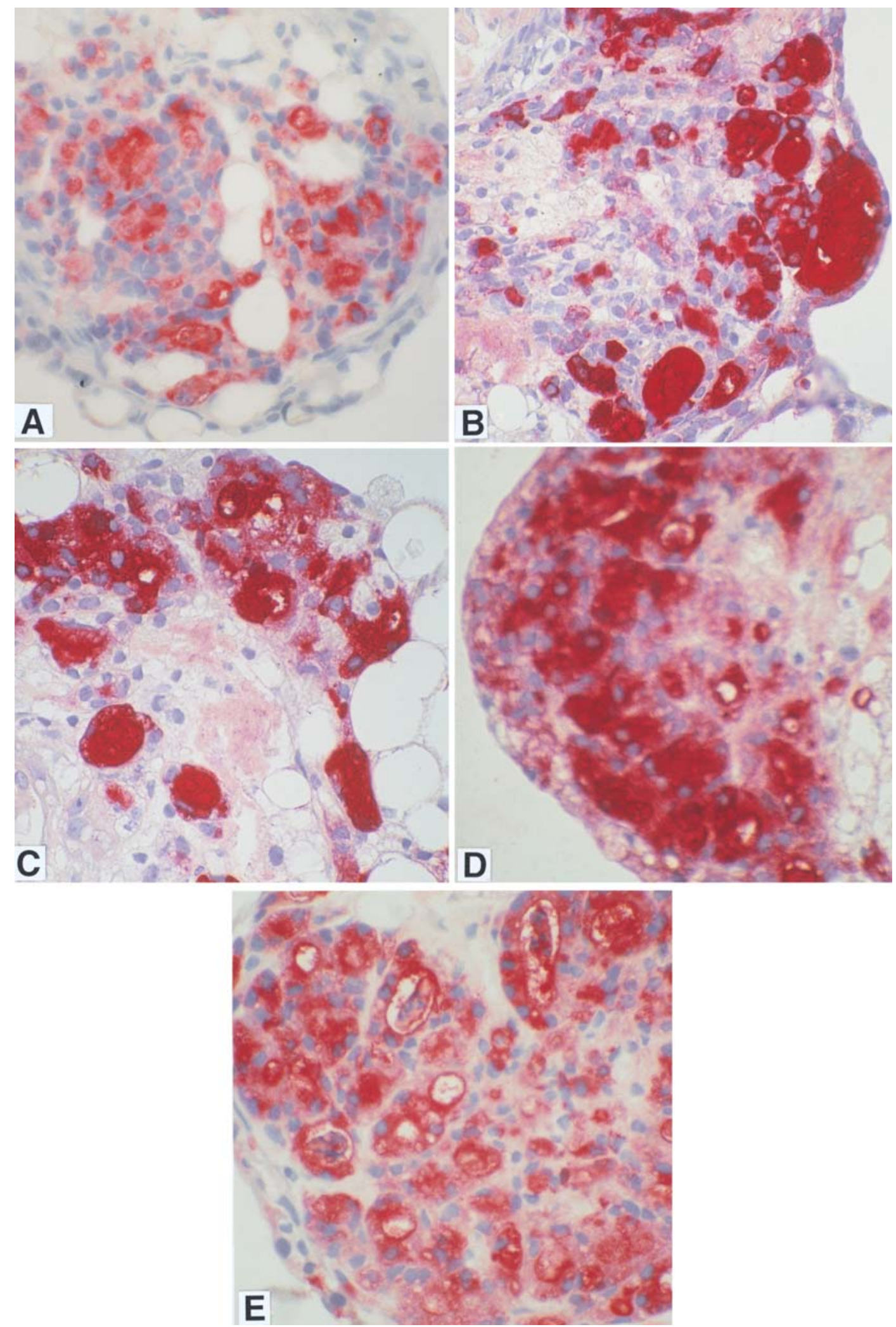

Fig. 1. Immunohistochemical staining for thyroglobulin in each of the treated groups: (A) Control group A, no supplements; (B) group B $(1 \mu M \mathrm{NaI})$; (C) group C (1 U/L TSH); (D) group D $(1 \mu M \mathrm{NaI} / 1 \mathrm{U} / \mathrm{L} \mathrm{TSH})$; and (E) group E $(1 \mu M \mathrm{NaI} / 1 \mathrm{U} / \mathrm{L} \mathrm{TSH}$ and $1 \mathrm{mM}$ MMI). In cultures treated with NaI (B) or NaI plus TSH (D), large follicles filled with thyroglobulin-containing colloid are present. In group E, which was treated with MMI in addition to NaI and TSH, follicles are small and the colloid is almost absent. 

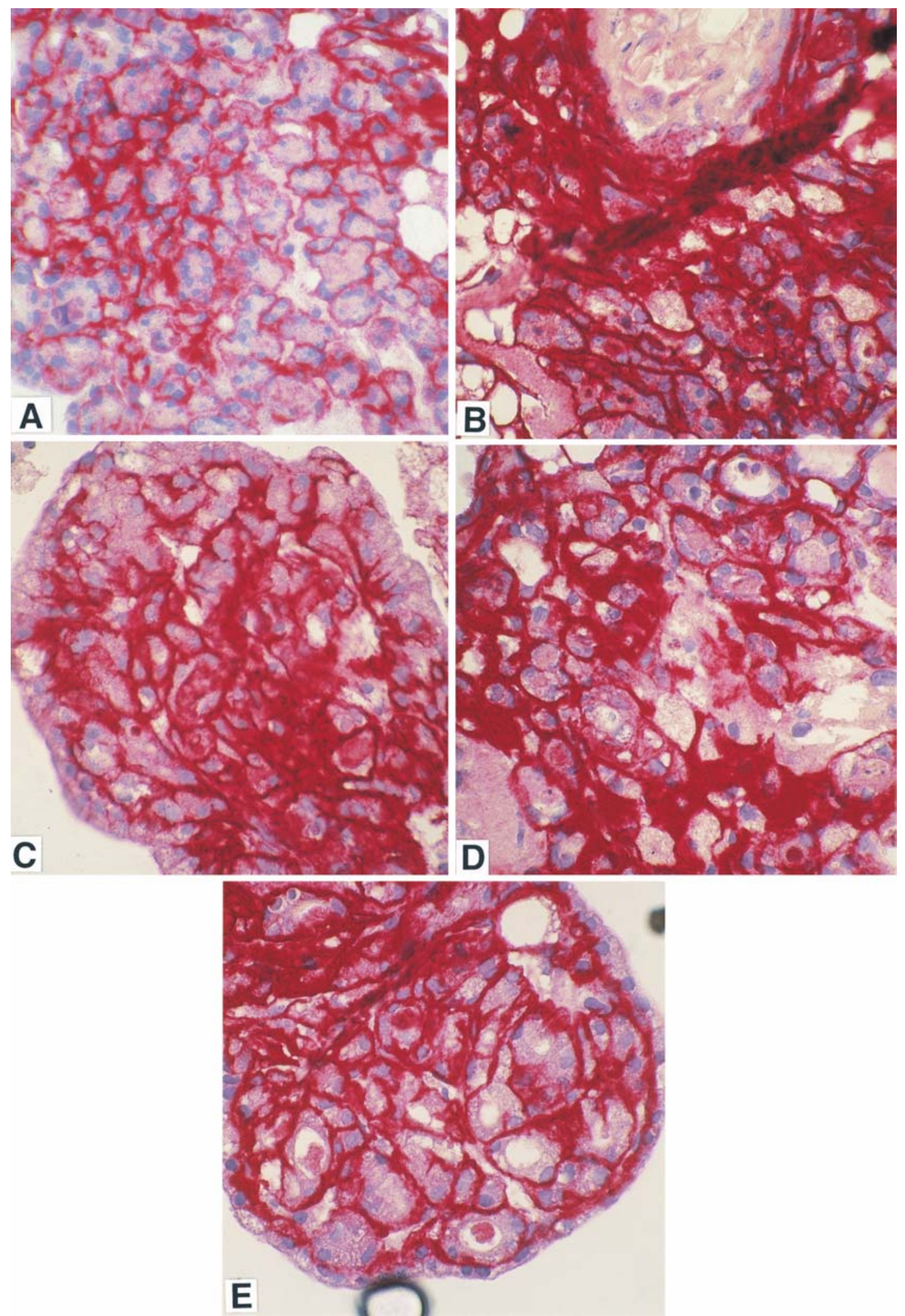

Fig. 2. Immunohistochemical staining for laminin in each of the tested groups: (A) Control group A, no supplements; (B) group B $(1 \mu M \mathrm{NaI})$; (C) group C (1 U/L TSH); (D) group D $(1 \mu M \mathrm{NaI} / 1 \mathrm{U} / \mathrm{L} \mathrm{TSH})$; and (E) group E $(1 \mu M \mathrm{NaI} / 1 \mathrm{U} / \mathrm{L} \mathrm{TSH}$ and $1 \mathrm{~m} M$ MMI). The BM component laminin is expressed and shows a normal distribution. However there is no obvious difference among the groups. 


\section{Materials and Methods}

\section{Culture of Thyroid Organoids}

Newborn Wistar rats weighing approx $25 \mathrm{~g}$ and not older than $48 \mathrm{~h}$ (from the Institute of Pathophysiology, University of Bern, Switzerland) were used. They were killed by $\mathrm{CO}_{2}$ exposure in a closed chamber and were then thyroidectomized under aseptic conditions. Five independent experiments with a total of 50 animals were performed. All animal experimentation was conducted with the highest standards of animal care, according to the ethical principles and guidelines of the Swiss Academy of Medical Sciences and approved by governmental authorities.

The thyroid tissue was cultured in a semisolid alginate bead system composed exclusively of polymerized alginate (Keltone LV, Kelco, Chicago, IL), which allows three-dimensional growth of follicles, cell clusters, and organoids, and itself contains no ECM components $(13,15)$. To initiate the cultures, each thyroid lobe was sliced into four pieces of about $1 \mathrm{~mm}$ side length, and the tissue was left in Coon's F-12 medium, supplemented with penicillin $(100 \mathrm{U} / \mathrm{mL})$, streptomycin $(100 \mu \mathrm{g} / \mathrm{mL})$, Lglutamine (200 nM) (Seromed, Biochrom KG, Berlin, Germany), 5\% newborn calf serum (Seromed), insulin (1.7 $\mu M)$, transferrin (55.6 $\mathrm{n} M)$, hydrocortisone $(8.8 \mathrm{n} M)$, glycin-L-histidyl-L-lysine (29.7 $\mathrm{n} M)$, and somatostatin (6.1 $\mathrm{n} M$ ) (Sigma Division of Fluka, Buchs, Switzerland) without TSH for $24 \mathrm{~h}$. The tissue was then incorporated into alginate beads approx $5 \mathrm{~mm}$ in diameter as previously described $(13,14)$. The beads were randomly distributed among five groups (A-E). Group A was the control group in which no additions were made to the medium. In groups B-E the culture medium was supplemented as follows: B, NaI (1 $\mu M)$; C, TSH (1U/L, Thytropar, Rover, Fort Washington, $\mathrm{PA}) ; \mathrm{D}, \mathrm{NaI}$ and TSH of the same concentrations as groups $\mathrm{B}$ and $\mathrm{C}$; E, NaI, TSH, and MMI (1 mM, Fluka AG, Buchs SG, Switzerland). The thyroid organoids were cultured for up to $21 \mathrm{~d}$, during which the culture medium was changed every 2 to $3 \mathrm{~d}$. In two of the five experiments, cell proliferation was documented by the incorporation of BrdU (Sigma, St. Louis, MO) into newly synthesized DNA during S-phase of the proliferating thyroid tissue in culture. BrdU can later be visualized in paraffin sections of cell clusters and organoids by indirect immunohistochemistry $(16,17)$. BrdU was added to the culture medium at a concentration of $0.06 \mathrm{mg} / \mathrm{mL}$ for the last $14 \mathrm{~h}$ before harvesting the cells.

At the end of the culture period, the beads were fixed in $4 \%$ phosphate-buffered formalin, $\mathrm{pH} 7.4$, containing $7 \mathrm{mM}$ $\mathrm{CaCl}_{2}$. The alginate was then dissolved in a sodium citrate buffer (150 m $M \mathrm{NaCl}, 55 \mathrm{~m} M$ Na citrate, $20 \mathrm{~m} M$ EDTA, $\mathrm{pH}$ 5.1). The cell clusters and organoids released were processed into a cell block using a Shandon cytoblock kit (Shandon, Pittsburgh, PA) and embedded in paraffin for further analyses, as previously described $(13,14)$.

\section{Morphological and Immunohistochemical Studies}

Two-micrometer paraffin sections were stained with periodic acid Schiff and hematoxylin for morphological and morphometric analyses. Follicle diameters were measured with a test screen (linear test circle series) according to Weibel (18). At least 58 follicles from each group were evaluated. For the first three experiments, photographs were taken of each section (magnification $\times 273$ ), and all follicles visible in the photographs were measured. For the last two experiments, a Visopan 314 projection microscope (Reichert-Jung, Vienna, Austria; magnification $\times 500$ ) was used with the same test screen as in the first three experiments. Mean and standard error of the mean (SEM) of follicular diameters were calculated (Table 1).

Alkaline phosphatase immunohistochemical staining of paraffin sections was used to study the distribution of ECM components. An indirect immunohistochemical method was performed as previously described (14) using rabbit polyclonal primary antibodies to EHS laminin (Sigma), human fibronectin (Dako A/S, Glostrup, Denmark), mouse collagen IV (Becton Dickenson), perlecan (kindly provided by Prof. J. Hassel, University of Pittsburgh, Pittsburgh, PA), and a swine antirabbit alkaline phosphatase-conjugated secondary antibody (Dako).

Thyroglobulin was also localized using the same method, with a rabbit polyclonal human thyroglobulin antibody (Dako) crossreacting with rat thyroglobulin and the same secondary antibody as just described.

BrdU was visualized using a triple antibody method with a mouse monoclonal bromodeoxyuridine antibody (Dako) followed by rabbit antimouse immunoglobulins (Dako) and finally mouse monoclonal alkaline phosphatase antialkaline phosphatase (Dako). The second and third steps were repeated, and the sections were then exposed to the substrate solution for $25 \mathrm{~min}$, as previously described (14). The slides were then rinsed overnight in running tap water, counterstained with hematoxylin, and sealed with Entellan (Merck, Darmstadt, Germany).

\section{Statistical Analyses}

Results of the measurements of follicular diameters are presented as means \pm SEM (Table 1). Statistical differences among the groups were tested by one-way analysis of variance using the Bonferroni/Dunn post-hoc test.

\section{Acknowledgments}

This work was supported by grants no. 31-34416.92 and 32-039355.93 from the Swiss National Science Foundation.

\section{References}

1. Marine, D. (1928) In: Comtes Rendues de la Conférence Internationale du Goiter 68-82, Hans Huber Verlag: Bern, Switzerland.

2. Marine, D. (1923) Western Reserve University Bulletin. Graham, W. (ed.) 7-123, Cleveland, OH. 
3. Astwood, E. B., Bissel, A., and Hughes, A. M. (1945) Endocrinology 37, 456-481.

4. Follis, R. H. Jr. (1959) Proc. Soc. Exp. Biol. Med. 100, 203-206.

5. Walthard, B. (1969) In: Spezielle Pathologische Anatomie. Doerr, W., Seifert, O., and Uehlinger, E. (eds.). Springer Verlag: New York.

6. Studer, H., Kohler, H., and Buergi, H. (1974). In: Thyroid. Handbook of Physiology. Greer, M. A. and Solomon, D. H., (eds.). American Physiological Society: Washington, DC.

7. Ramelli, F., Studer, H., and Bruggisser, D. (1982). Am. J. Pathol. 109, 215-223.

8. Studer, H., and Gerber, H. (1991). In: Werner's and Ingbar's, The Thyroid. Braverman, L. E. and Utiger, R., (eds.). JB Lippincott: Philadelphia.

9. Gerber, H., Studer, H., Conti, A., Engler, H., Kohler, H., and Haeberli, A. (1981). J. Clin. Invest. 68, 1338-1347.

10. Gerber, H., Huber, G., Peter, H. J., Kämpf, J., LemarchandBeraud, T., Fragu, P., and Stocker, R. (1994). Endocrinology 135, 2688-2699.
11. Tsukui, T., Aizawa, T., Yamada, T., and Kawabe, T. (1978). Endocrinology 102, 1662-1669.

12. Hay, E. D. (1991). Cell Biology of the Extracellular Matrix, 2nd ed., Plenum: New York.

13. Bürgi-Saville, M. E., Gerber, H., Peter, H. J., Paulsson, M., Aeschlimann, D., Glaser, C., Kämpf, J., Ruchti, C., Sidiropoulos, I., and Bürgi, U. (1997). Thyroid 7, 347-356.

14. Bürgi-Saville, M. E., Reut, B., Gerber, H., Peter, H. J., Paulsson, M., Kämpf, J., Simon, F., Marti, U., and Bürgi, U. (1998). Thyroid 8, 1147-1155.

15. Hauselmann, H. J., Aydelotte, M. B., Schumacher, B. L., Kuettner, K. E., Gitelis, S. H., and Thonar, E. J. (1992). Matrix 12, 116-129.

16. Ellwart, J. and Dormer, P. (1985). Cytometry 6, 513-520.

17. Magaud, J. P., Sargent, I., Clarke, P. J., French, M., Rimokh, R., and Mason, D. Y. (1989). J. Histochem. Cytochem. 37, 1517-1527.

18. Weibel, E. R. (1979). Practical Methods for Biological Morphometry, vol. 1. Academic: London. 